2000. $-510 \mathrm{c}$.

3. Фридман Д. Ордуэй. Анализ и оценка приносящей доход недвижимости. М.: Дело, 1995. 542 с.

УДК 69.003

ББК 65.31

Исмаилов Р.Т., Наврузбекова Н.Ф.

СОВЕРШЕНСТВОВАНИЕ МЕТОДИЧЕСКИХ ОСНОВ КОМПЛЕКСНОГО ЭКОНОМИЧЕСКОГО АНАЛИЗА СТРОИТЕЛЬНОГО ПРЕДПРИЯТия

Ismailov R.T., Navruzbevova N.F.

\title{
IMPROVEMENT METHODICAL BASES COMPLEX ECONOMIC ANALY- SIS OF THE BUILDING ENTERPRISE
}

Рассматривается и уточняется содержание и взаимосвязь основных этапов проведения комплексного экономического анализа внешней и внутренней среды строительного предприятия как основного средства для получения недостающей для принятия решений информаџии, всесторонне, полно и иелостно отражающей состояние и эффективность его хозяйственной деятельности. Предлагаются принципь реализаџии таких основных свойств экономического анализа как системность, иеленаправленность, избирательность и достоверность получаемой на его основе информачии.

Ключевые слова: строительное предприятие, комплексный экономический анализ, взаимосвязь этапов проведения анализа, динамический анализ.

It is considered and elaborated contents and intercoupling main stage undertaking the complex economic analysis external and internal ambience of the building enterprise as the main means for reception lacking for decision making of information, all-round, packed and holistic reflecting condition and efficiency to economic activity of the building enterprise. They are offered principles to realization such main characteristic of the economic analysis as systematic, centrality of purpose, selectivity and validity got on his base of information.

Key words: building enterprise, complex economic analysis, intercoupling stage undertaking the analysis, dynamic analysis.

Экономический анализ является одним из основных средств исследования и оценки состояния строительного предприятия и его окружающей среды, проводимого с цель формирования информационного обеспечения для эффективного управления поведением в изменяющихся условиях современного рынка. Следовательно, в нестабильных условиях функционирования, развитие экономического анализа путем повышения уровня его комплексно- 
сти, системности, широкого использования экономико-математических методов и ПЭВМ является одной из актуальных проблем экономической науки.

Хозяйственная деятельность строительного предприятия является многоплановой, а в условиях нестабильного рынка еще и слабо предсказуемой. Она исследуется разнообразными специалистами с различных сторон и точек зрения. Результаты такого исследования должны быть представлены в виде конкретных знаний о различных закономерностях хозяйственной деятельности строительного предприятия и достаточными для решения различных производственных и хозяйственных задач. Однако в условиях современной модернизации экономики на инновационной основе чрезвычайно усложнилось взаимодействие строительных предприятий с внешней средой. Поэтому повышение эффективности производства, улучшение качества производимой продукции, решение социальных и целого ряда других проблем на строительном предприятии требует комплексных, т. е. всесторонних знаний о его фактическом состоянии, а также тенденциях и перспективах дальнейшего развития.

Такие знания не могут быть получены в результате простого суммирования тех конкретных сведений, которыми располагают различные специалисты, занятые, например, планированием, организацией, учетом производства и другими видами функциональной и управленческой деятельности. Необходимо, чтобы конкретные сведения о различных сторонах хозяйственной деятельности были органически увязаны между собой исходя из единой цели и задач проводимого исследования.

Другими словами, проводимый анализ должен быть целенаправленным и ориентированным на решение как текущих проблем стоящих перед строительным предприятием, так и проблем, которые необходимо будет решать в будущем, отвечая на различные вызовы рыночной среды. Только в этом случае данные, получаемые в результате анализа будут представлять собой единую комплексную систему знаний, которую действительно можно назвать эффективным информационным ресурсом, представляющим собой важнейшее средство для решения сложных хозяйственных и управленческих задач [1].

Таким образом, комплексный экономический анализ выступает как средство получения недостающей для принятия решений информации, всесторонне, полно и целостно пополняющей и отражающей данные о состоянии и эффективности хозяйственной деятельности строительного предприятия.

Комплексный анализ предполагает изучение всех сторон производственнохозяйственной деятельности строительного предприятия. Но для удовлетворения требованиям комплексности экономического анализа недостаточно определения и полноты охвата состояния строительного предприятия, т. е. анализа всех сторон его производственно-хозяйственной деятельности. Необходим полный и всесторонний анализ всех процессов деятельности строительного предприятия, протекающих как во внутренней, так и в его окружающей среде.

Другим необходимым условием достижения комплексности анализа является определение в нем единой цели, позволяющей объединять отдельные направления исследования и анализа показателей и факторов производства в целостную систему изучения хозяйственной деятельности строительного 
предприятия и его окружающей среды. Единая цель выступает организующим началом, обеспечивающим согласование результатов экономического анализа предприятия в целом с результатами экономического анализа отдельных его частей или сторон. Единство цели превращает комплексный анализ в вид системного анализа. Комплексность и системность как важнейшие принципы экономического анализа взаимосвязаны между собой и дополняют друг друга [2].

Помимо целесообразности комплексный анализ должен обладать и свойством избирательности и достоверности, получаемой на каждом его этапе информации. Необходимость избирательности вытекает из условия целесообразности, т.е. в процессе проведения анализа на каждом его шаге должна обрабатываться только та информация, которая непосредственно связана с текущей решаемой проблемой.

Характерной особенностью функционирования строительного предприятия в условиях рынка является быстрая смена внешних условий его функционирования. Следовательно, комплексный анализ итогов деятельности строительного предприятия по истечению заданного отчетного периода не позволяет организовать эффективное управление строительным производством в реальном времени. Таким образом, комплексный экономический анализ в условиях рынка должен обладать еще и свойством динамичности или возможностью наблюдать и оценивать эффективность функционирования строительного предприятия во времени. Это дает возможность менеджменту предприятия принимать не только эффективные, но и своевременные хозяйственные и управленческие решения и тем самым, повысить эффективность управления строительным производством в условиях рынка. Для реализации комплексного динамического анализа строительного предприятия необходима соответствующая система показателей, позволяющая отражать динамику протекающих во времени производственных процессов. На наш взгляд, для этой цели в дополнение к известным статическим показателям можно использовать такой динамический показатель, как скорость их изменения во времени, которая периодически пересчитывается по мере изменения условий функционирования строительного предприятия. Для оценки текущей динамики различных показателей можно воспользоваться следующим соотношением:

$$
\Delta \Pi=\left(\Pi_{2}-\Pi_{1}\right) /\left(T_{2}-T_{1}\right),
$$

где $\Pi_{1}$ и $\Pi_{2}$ - значения анализируемого показателя соответственно вычисленные для моментов времени $T_{1}$ и $T_{2}, T_{2}>T_{1}$.

Таким образом, необходим комплексный подход к проведению анализа хозяйственной деятельности предприятия, сочетающий в себе как анализ текущей деятельности строительного предприятия, или оперативный анализ, так и анализ итогов деятельности строительного предприятия за отчетный период или итоговый анализ. При этом комплексный анализ должен опираться на данные мониторинга окружающей среды строительного предприятия, для реализации которого строительному предприятию необходимо иметь корпоративную и локальную вычислительные сети, позволяющие оперативно осуществлять сбор и передачу данных в аналитический центр строительного 
предприятия, занимающийся проведением комплексного анализа.

В общем случае, взаимосвязи различных блоков показателей работы строительного предприятия, определяющие последовательность этапов проведения комплексного экономического анализа можно отразить в виде следующей структуры (рисунок 1).

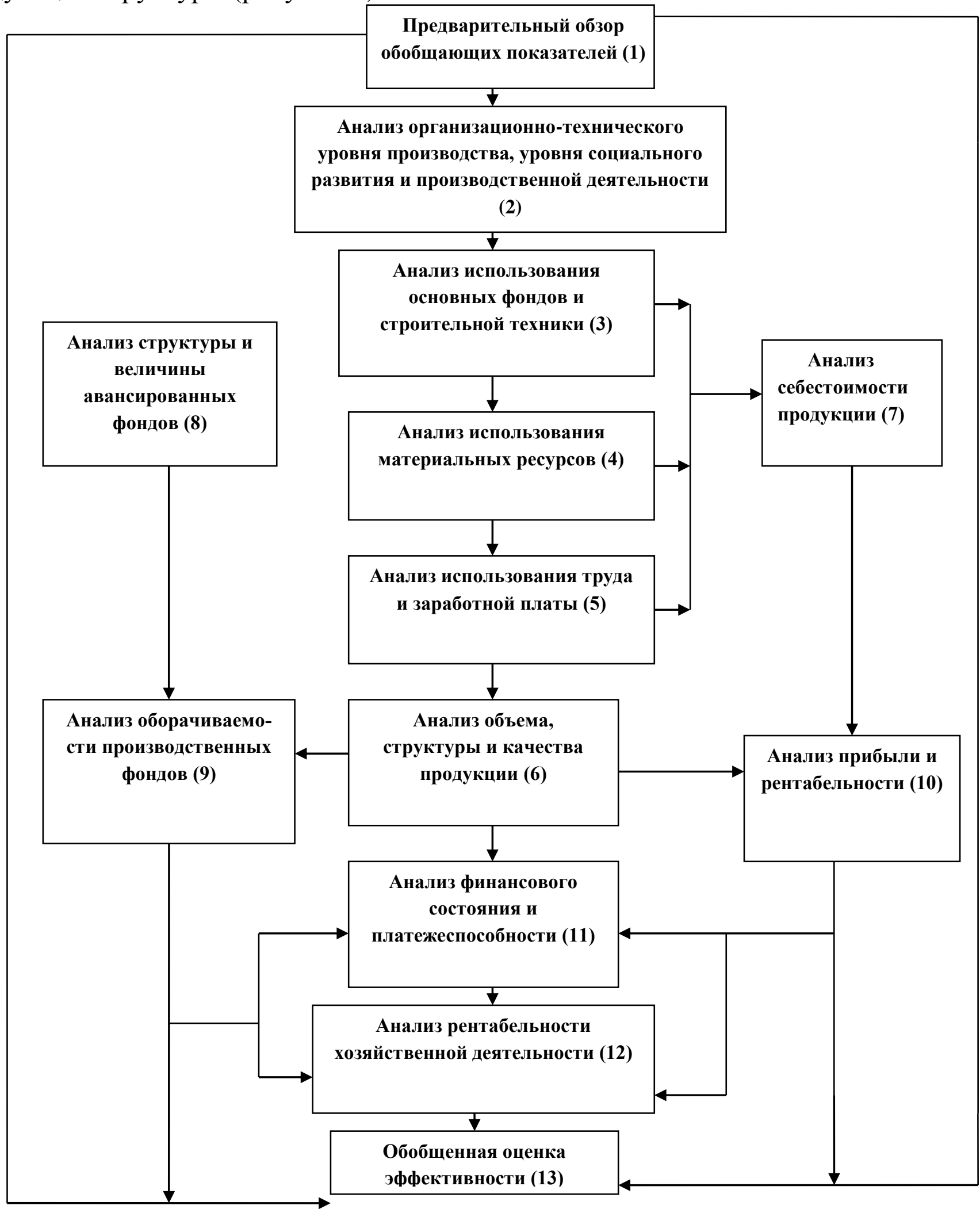

Рисунок 1 - Взаимосвязь различных блоков показателей работы строительного предприятия 
Блок I «Предварительный обзор обобщающих показателей», осуществляется по основным показателям, характеризующим общие результаты и эффективность производственно-хозяйственной деятельности предприятия. Такой обзор проводится для систематизации обобщающих показателей и действующих на них факторов без раскрытия их внутреннего содержания.

Его задача заключается в общей характеристике работы предприятия по выполнению повышенных плановых заданий и обязательств, а также в определении динамики показателей по сравнению с предшествующими периодами.

Блок 2 «Анализ организационно-технического уровня и других показателей производства». Совокупность показателей, относящихся к блоку 2, определяет степень использования производственных ресурсов (производительность труда, фондоотдачу основных производственных фондов и материалоотдачу предметов труда), а также другие показатели экономической эффективности: качество, себестоимость и прибыльность строительной продукции, оборачиваемость производственных фондов, рентабельность производственнохозяйственной деятельности и финансовое состояние.

Блок 3 «Анализ использования основных фондов и оборудования». Основными синтетическими показателями состояния и использования средств труда являются: итоговые значения и динамики изменения текущей фондоотдачи или фондоемкости; средняя стоимость основных производственных фондов строительного предприятия и амортизационные отчисления.

Через выходные показатели блока 3 устанавливается его связь с блоками 6 , 7 и 8. Наряду с этими показателями, большое значение имеют и другие, например, капитальные вложения и ввод в действие основных производственных фондов, внедрение новой техники и т.д. Первичным показателем интенсивности использования основных фондов является производительность или выработка строительной техники, определяемая объемами выполненных с ее помощью подрядных работ.

Общий показатель фондоотдачи основных производственных фондов является функцией от показателя интенсивности использования строительной техники (часовая выработка продукции с помощью действующей строительной техники) и ряда показателей экстенсивного использования строительной техники и фондов (коэффициент использования времени действующей строительной техники, коэффициент использования строительной техники и оборудования, доля строительной техники и оборудования в общей стоимости основных производственных фондов).

Бло к 4 «Анализ использования материальных ресурсов». Основными выходными показателями состояния и использования предметов труда являются: материалоотдача, ее динамика, или выход продукции, в расчете на каждый рубль стоимости предметов труда в единицу времени, обратным ему показателем является материалоемкость; стоимость израсходованных предметов труда за отчетный период.

Наряду с этими показателями анализируются и другие аналитические показатели, например, выполнение задания по среднему снижению норм расхода важнейших материальных ресурсов и т. д. Классификация показателей и 
факторов использования предметов труда позволяет наметить пути выявления резервов использования предметов труда применительно к особенностям строительной отрасли.

Бл о к 5 «Анализ использования труда и заработной платы. Основными выходными показателями этого блока являются следующие: средняя производительность труда одного работающего и скорость ее динамики; средняя численность работающих; общий фонд оплаты труда производственного персонала; средняя заработная плата одного работающего и динамика ее изменения.

Средняя производительность труда одного работающего является функцией от показателя среднечасовой производительности труда, в наибольшей степени характеризующего интенсивные факторы использования труда, и ряда показателей экстенсивного использования труда (количество рабочих часов в дне и рабочих дней в периоде, удельный вес рабочих в составе производственного персонала).

Следует отметить, что научно обоснованная организация производственного процесса требует пропорциональности используемых ресурсов. Объем производства лимитируется теми ресурсами, наличие которых минимально. Недостаток производственных ресурсов может быть восполнен за счет улучшения качественных показателей.

Характер участия отдельных элементов процесса труда в производственном процессе различен. Только труд является источником вновь созданной стоимости. Но в процессе труда часть стоимости средств труда и стоимость используемых предметов труда переносятся на стоимость произведенной продукции.

Блок 6 «Анализ объема, структурь и качества продукции, работ и услуг». Обобщающим показателем этого блока является стоимость реализованной товарной продукции, работ и услуг в рыночных ценах. Это выходной синтетический показатель, который является функцией количества произведенной и проданной строительной продукции, ее структуры и качества.

Блок 7 «Анализ себестоимости продукции». Выходным синтетическим показателем этого блока является полная себестоимость реализованной товарной продукции. Большое значение имеют и такие показатели, как полная себестоимость товарной продукции, себестоимость валовой продукции, общие затраты на производство и др.

Связь данного блока с блоками 3, 4 и 5 осуществляется через показатели амортизационных отчислений, затрат предметов труда на производство и фонд заработной платы.

Блок 8 «Анализ величины и структуры авансированных фондов». Основными синтетическими показателями этого блока являются: средняя стоимость основных производственных фондов; средняя стоимость оборотных средств, в том числе нормируемых; сумма собственных средств предприятия, в том числе собственных оборотных средств.

Блок 9 «Анализ оборачиваемости производственных фондов». Основные синтетические показатели этого блока следующие: оборачиваемость ос- 
новных производственных фондов; фондоотдача основных производственных фондов; оборачиваемость оборотных средств; оборачиваемость нормируемых оборотных средств; общая фондоотдача производственных фондов.

Блок 10 «Анализ прибыльности и рентабельности произведенной продукции». Результат производственно - хозяйственной деятельности аккумулируется в показателе балансовой прибыли. Для оценки результатов труда коллектива предприятия определяют так называемую расчетную прибыль. Показателями рентабельности реализованной продукции являются: удельная прибыль; отношение прибыли к себестоимости; затраты на один рубль реализованной товарной продукции.

Эти показатели рассчитывают как по всей товарной или реализованной товарной продукции, так и по отдельным изделиям или группам изделий.

Блок 11 «Анализ финансового состояния и платежеспособности». Оборачиваемость производственных фондов (блок 9) является важнейшим фактором финансового состояния предприятия, которое в основном определяется соотношением величин запасов нормируемых оборотных средств, собственных оборотных средств и заемных средств, предназначенных для формирования запасов оборотных средств. Величина собственных оборотных средств определяется во многом пополнением оборотных средств за счет прибыли. Через ту часть прибыли, которая предназначена для пополнения собственных оборотных средств, осуществляется связь и влияние блока 10 на блок 11.

Блок 12 «Анализ рентабельности хозяйственной деятельности». Одним из показателей, рентабельности производственно-хозяйственной деятельности является отношение прибыли от реализованной продукции к фондам. Этот показатель исчисляется умножением удельной прибыли (блок 10) на общую фондоотдачу производственных фондов (блок 9).

Показатели рентабельности производственно-хозяйственной деятельности иногда называют рентабельностью производственных фондов (фондорентабельностью), так как эти показатели можно рассчитать на основе данных о прибыли (блок 10) и величине производственных фондов (блок 8).

Блок 10 охватывает показатели, характеризующие формирование прибыли, а блок 11 - распределение прибыли. Причем процесс распределения прибыли активно воздействует на улучшение результативных показателей производственно-хозяйственной деятельности.

Блок 13 «Обобщцющая оценка эффективности работы и анализ экономического стимулирования". Важное место в системе комплексного экономического анализа занимает оценка хозяйственной деятельности, представляющая собой общий вывод о результатах деятельности на основе качественного и количественного анализа хозяйственных процессов, отражаемых системой показателей. Оценка деятельности строительного предприятия проводится на первом этапе комплексного экономического анализа, когда определяются основные направления аналитической работы (предварительная оценка), и на заключительном этапе, когда подводятся итоги анализа (окончательная оценка). Окончательная оценка является важным информацион- 
ным источником для обоснования и принятия оптимального управленческого решения в конкретной ситуации.

Для объективной оценки работы показатели хозяйственной деятельности корректируются по результатам анализа: вычитаются (или прибавляются) суммы, полученные в результате действия внешних факторов (изменение цен, тарифов и т. д.), нарушений государственной и хозяйственной дисциплины. Очищенные отчетные показатели полнее характеризуют хозрасчетную деятельность производственных коллективов, принимаются за базу расчета фондов экономического стимулирования.

Экономическое стимулирование хозяйственной деятельности на предприятии включает несколько основных подсистем: материального стимулирования; морального стимулирования; экономического воздействия на производство (финансы, кредит, ценообразование и др.).

Анализ эффективности материального стимулирования должен включать анализ следующих компонентов: эффективности организации заработной платы; эффективности систем премирования; эффективности организации материальной ответственности.

Следует заметить, что рассмотренная структура организации комплексного экономического анализа ориентирована на исследование внутренней среды строительного предприятия и не затрагивает проблемы его взаимодействия с окружающей средой. Учитывая существенное влияние внешней среды на эффективность функционирования строительного предприятия в условиях рынка комплексный экономический анализ должен предусматривать и проведение анализа основных составляющей внешней среды строительного предприятия. На наш взгляд, комплексный анализ внешней среды строительного предприятия может быть реализован согласно представленной структуре организации его проведения (рисунок 2).

В рассмотренную схему не вошел блок анализа взаимодействия строительного предприятия с финансовыми и государственными учреждениями, которые определяются соответственно кредитно-денежными отношениями предприятий с банками и нормами, установленными в законодательном порядке. В целом же приведенная схема достаточно полно отражает взаимодействие различных субъектов рынка строительной продукции.

1. Мониторинг окружающей среды сводится к сбору и первичной переработки информации, отражающей ее текущее состояние и перспективы развития с целью определения наличия в ней факторов, влияющих на строительное предприятие и на его взаимодействие с различными субъектами рынка.

2. Анализ конкурентоспособности строительного предприятия. Этот блок связан со сбором информации об основных конкурентах, с целью определения имеющихся у исследуемого предприятия устойчивых конкурентных преимуществ и определения путей повышения его конкурентоспособности на рынке.

3. Анализ поставщиков материальных ресурсов. Данный блок предназначен для получения информации, характеризующей потенциальные возможно- 
сти рынка строительных материалов, машин и механизмов и перспектив его развития. Полученные таким образом данные используются для определения внешних условий и возможностей строительного предприятия, способствующих развитию его производственного потенциала.

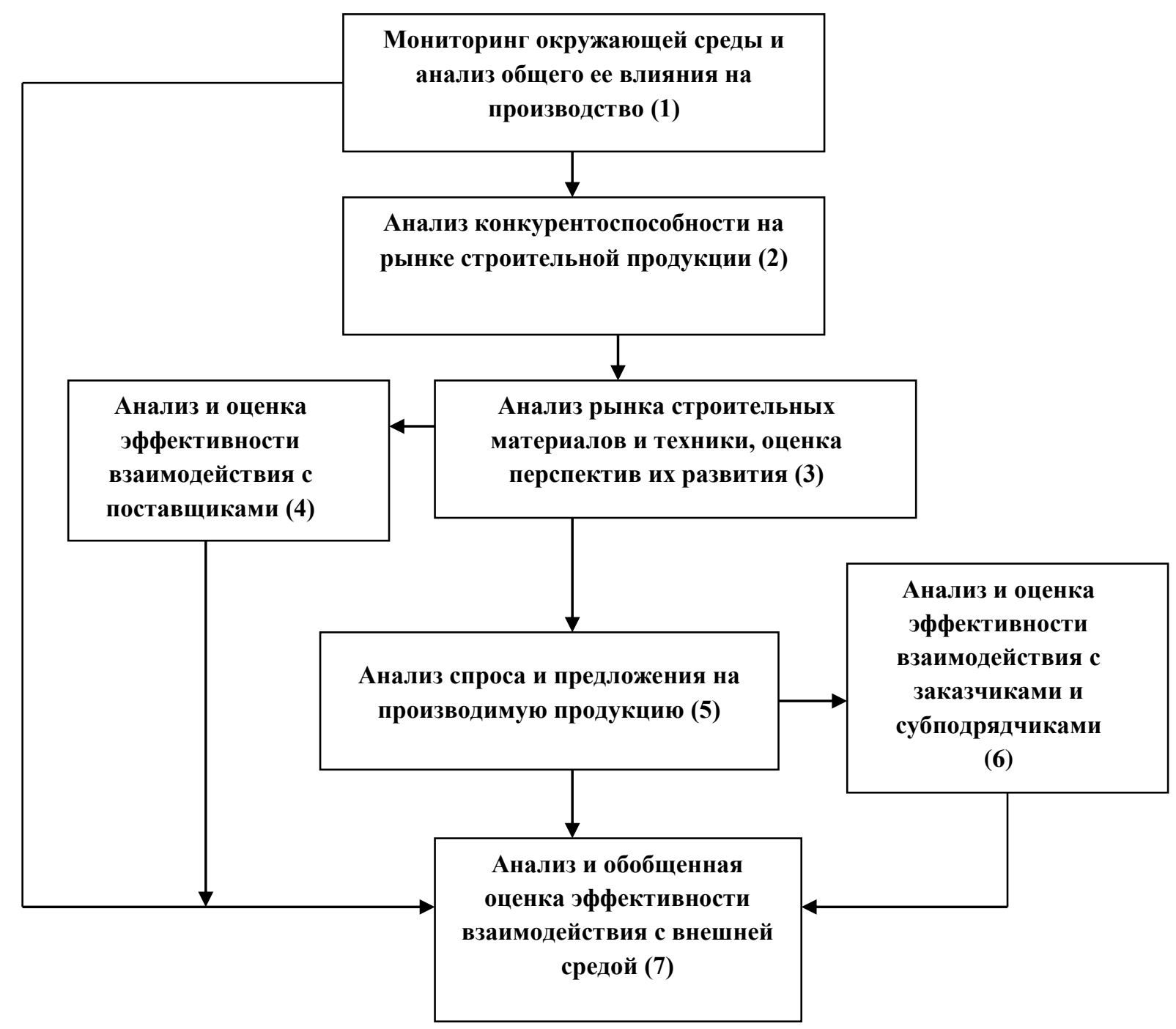

Рисунок 2 - Структура комплексного анализа взаимодействия строительного предприятия с внешней средой

4. Анализ эффективности взаимодействия с поставщчиками. Этот блок отвечает за сбор информации, позволяющей оценить надежность поставщиков и то, насколько взаимодействие с ними носит оптимальный характер. В задачи этого блока входит также сбор информации о потенциальных поставщиках новых строительных материалов и новой строительной техники.

5. Анализ спроса и предложения. Задача этого блока заключается в проведении анализа спроса и предложения на различные виды строительной продукции на различных сегментах рынка. Этот блок также отвечает за определение наиболее перспективных видов строительной продукции, как в ближайшее время, так и в отдаленной перспективе. 
6. Анализ взаимодействия с субподрядчиками заказчиками. В данном блоке осуществляется переработка и анализ информации, позволяющей определить целесообразность заключения контрактов с наиболее эффективно функционирующими специализированными предприятиями, способными качественно выполнить работы, не свойственные строительному предприятию общего назначения. Здесь также проводится анализ потребительских характеристик потенциальных заказчиков, их основных требований и покупательской способности.

7. Анализ и обобщенная оценка взаимодействия с внещней средой. Этот блок предназначен для обобщения полученных аналитических данных и проведения общей оценки эффективности взаимодействия строительного предприятия с внешней экономической средой.

В заключении следует отметить, что предложенные схемы позволяют строительному предприятию организовать комплексный анализ внутренней и внешней среды в нестабильных условиях современного рынка.

\section{Библиографический список:}

1. Селезнева Н.Н., Ионова А.Ф. Финансовый анализ. - М.: ЮНИТИ-ДАНА, 2006. $-638 \mathrm{c}$.

2. Экономический анализ: ситуации, тесты, примеры / Под ред. Баканова М.И., Шеремета А.Д. -М.: Финансы и статистика, 2003 .-656 с.

УДК 69.003.

ББК 65.31

Магомедов А.Г., Зейналов М.3.

УПРАВЛЕНИЕ РАЗВИТИЕМ МОЩНОСТИ ОСНОВНЫХ ПРОИЗВОДСТВЕННЫХ ФОНДОВ СТРОИТЕЛЬНОГО ПРЕДПРИЯТИЯ

Magomedov A.G., Zeynalov M.Z.

\section{MANAGEMENT DEVELOPMENT TO POWERS OF THE PRODUCTION CAPITAL FUND OF THE BUILDING ENTERPRISE}

В статье рассматриваются проблемы оценки и управления развитием мощности основных фондов строительного предприятия. Проводится критический анализ способов оценки мощности основных фондов, предложенных различныли авторами, и на этой основе совершенствуется способ, связанный с выбором ведущего звена. Предложена методика, позволяющая регулировать структуру основных фондов строительного предприятия с учетом имеющейся у него строительной техники.

Ключевые слова: мощчность основных фондов, оченка мощности, однотипные подрядные работы, регулирование структуры основных фондов. 